\title{
Barreiras para atuação do profissional de educação física na atenção primária à saúde
}

\author{
Barriers to physical activity professional acts in primary health care \\ Barreras para la actuación del profesional de educación física em la atención primaria em \\ salud
}

Cinthya Rafaella Magalhães da Nóbrega Novaes ${ }^{1,2 *}$, Flávia Accioly Canuto Wanderley¹, Maria Roseane Alves da Silva¹, Bárbara Marcelly Tavares de Gusmão¹.

\section{RESUMO}

Objetivo: Identificar as barreiras para atuação do profissional de Educação Física na atenção primária em saúde. Métodos: Para revisão integrativa da literatura foram realizadas buscas na Biblioteca Virtual em Saúde, nas bases Literatura Latino-Americana e do Caribe em Ciências da Saúde (LILACS) e Scientific Electronic Library Online (SCIELO), utilizando os descritores: saúde pública, promoção da saúde, atenção primária à saúde, prática profissional, educação física e treinamento. Foram incluídos documentos na íntegra, publicados a partir de 2005 em português e/ou inglês. Resultados: Catorze artigos foram inclusos. Identificouse como barreiras: formação profissional, deficiência da estrutura física e recursos materiais, representação social de gestores, profissionais e usuários sobre Educação Física, número reduzido de profissionais atuando e dificuldade para o trabalho interdisciplinar. Considerações finais: As principais barreiras para atuação do profissional de educação física na atenção básica estão relacionadas com a gestão dos programas onde esses profissionais atuam e com a formação deste profissional.

Palavras-chave: Saúde pública, Promoção da saúde, Atenção primária à saúde, Educação física e treinamento, Prática profissional.

\begin{abstract}
Objective: The study aimed to identify the perceived barriers to physical activity professional act in Primary Health Care. Methods: An integrative review was conducted in Biblioteca Virtual em Saúde, Literatura LatinoAmericana e do Caribe em Ciências da Saúde (LILACS) and Scientific Electronic Library Online (SCIELO) bases. The selected key words were: public health, health promotion, Primary Health Care, professional practice, Physical education and training. The inclusion criteria were: the article must be full-text available, it has to be published after 2005 in Portuguese or English language. Results: After analysis, fourteen articles were included. It was observed that academic formation is the most evident barrier to physical activity professional acts in Primary Health Care. The Lack of infrastructure and materials, inadequate perception of physical activity professional relevance by managers, other health professionals and general population, few professionals to attend population and lack of interdisciplinarity were also mentioned as barriers. Final considerations: The greater barriers to the performance of physical education professionals in primary health care are related to the management of the programs where these professionals work and to their graduation process.
\end{abstract}

Keywords: Public health, Health promotion, Primary health care, Physical education and training, Professional practice.

1 Universidade Estadual de Ciências da Saúde (UNCISAL), Maceió - AL.

${ }^{2}$ Laboratório de Cineantropometria, atividade física e promoção da saúde (LACAPS/UFAL), Arapiraca - AL.

*E-mail: rafamn20@gmail.com 


\section{RESUMEN}

Objetivo: El estudio buscó identificar las barreras para el desempeño de los profesionales de Educación Física en la atención primaria de salud. Métodos: Para una revisión bibliográfica integradora de la literatura se realizaron búsquedas en la Biblioteca Virtual de Salud, en las bases de datos en línea de Literatura Latinoamericana y del Caribe en Ciencias de la Salud (LILACS) y Biblioteca Electrónica Científica (SCIELO), utilizando los descriptores: salud pública, promoción de la salud, atención primaria a la salud, práctica profesional, educación física y entrenamiento. Se incluyeron documentos en su totalidad, publicados desde 2005 en portugués y / o inglés. Resultados: Se incluyeron catorce artículos. Se identificaron las siguientes barreras: capacitación profesional, deficiencia en la estructura física y recursos materiales, representación social de gerentes, profesionales y usuarios sobre educación física, un número reducido de profesionales que trabajan y dificultades en el trabajo interdisciplinario. Consideraciones finales: Los resultados muestran que algunas de las barreras para el desempeño de los profesionales de educación física en atención primaria están relacionadas con la gestión de los programas donde trabajan estos profesionales y con la formación de este profesional.

Palabras clave: Salud pública, Promoción de la salud, Atención primaria a la salud, Educación física y entrenamiento, Práctica profesional.

\section{INTRODUÇÃO}

De acordo com a Organização Mundial de Saúde (OMS), a inatividade física se caracteriza atualmente como o quarto fator de risco para doenças crônicas não transmissíveis (WHO, 2010). Estima-se que 6\% das mortes ocorridas no mundo estão relacionadas a inatividade física, e que a redução da prevalência deste fator de risco em $10 \%$ a 25\% poderia impactar na redução de mais de 1,3 milhões de óbitos (LEE JM, et al 2012).

Considerando o avanço tecnológico e o fenômeno de transição epidemiológica ocorrido na última década, Hamilton MT, et al (2012) chamam atenção para o comportamento sedentário, que não diz respeito exclusivamente à baixos níveis ou inexistência de hábitos que envolvam atividade física na rotina, mas considera também a associação destes a outros hábitos de baixo nível de gasto energético que, combinados entre si, podem comprometer o estado geral de saúde dos indivíduos. São exemplos desses hábitos: o tempo sentado, o tempo de uso de aparelhos de tela e o transporte inativo.

A prática regular de atividade física está associada com melhores condições de saúde e de qualidade de vida. Atualmente está cada vez mais clara a importância da manutenção de um estilo de vida ativo para a saúde da população em geral (GROPPO HS, et al., 2012; HALLAL PC, et al., 2010; MENDONÇA BCA, et al., 2009). Desse modo, o crescimento de iniciativas, programas e campanhas a favor de um estilo de vida mais ativo vem ganhando maior importância, expressão e reconhecimento (HALLAL PC, et al., 2010).

Apesar de um corpo de evidências significativo comprovar os benefícios da atividade física para promoção da saúde, a população mundial é cada vez menos ativa fisicamente (PITANGA FJG e LESSA I, 2005; JESUS GM e JESUS EFA, 2012). Para enfrentar esse quadro de baixos níveis de atividade física, em 2006 foi aprovada a Política Nacional de Promoção da Saúde (PNPS) que inseriu a prática de atividade física na estratégia de promoção de saúde, reforçando a importância do estilo de vida fisicamente ativo (PINTO NRS, et al., 2009).

Em 2008, para atuar nessa estratégia o Profissional de Educação Física (PEF) foi inserido no Sistema Único de Saúde (SUS) nas equipes do Núcleo de Apoio à Saúde da família (NASF), Academia da Cidade e em projetos que visam a promoção, proteção e reabilitação da saúde. Ainda em 2008, através da portaria 154, de 24 de janeiro, esses profissionais foram formalmente inseridos na Atenção Primária a Saúde - APS (BRASIL, 1990; BRASIL, 2008).

A PNPS evidenciou a importância da prática de atividade física pela população em geral e por outro lado, a necessidade de PEF qualificados para atuar nesse novo campo de trabalho, tendo em vista que a atuação adequada e eficiente desses profissionais na APS potencializa os resultados esperados em busca de maiores 
níveis de atividade física da população. Além disso, sua prática regular previne doenças crônicas não transmissíveis (DCNT) como diabetes, hipertensão e alguns tipos de câncer, melhorando ainda mobilidade e capacidade funcional dos indivíduos no envelhecimento (MATSUDO SM, 2006).

A inserção deste profissional na APS é vista como um fator favorável tanto para o serviço de saúde quanto para a categoria profissional (FALCI DM e BELISÁRIO SA, 2013). Todavia há evidências de que o potencial de promoção da atividade física proveniente da atuação do PEF deve ser melhor explorado, com vista à superação da perspectiva reducionista de que suas práticas laborais tem como função prioritária a minimização dos impactos de uma vida sedentária (CARVALHO AS, et al., 2017).

Estudos têm demonstrado as perspectivas da atuação do PEF no Sistema Único de Saúde (SUS), contudo, muitos desfechos apontam que estes profissionais estão limitados à realização de apenas alguns tipos de intervenções, como: caminhadas, alongamentos e ações envolvendo a população idosa ou grupos específicos como hipertensos e diabéticos numa perspectiva curativista (CARVALHO AS, et al., 2017).

Essa atuação ainda tímida e sem contemplar o que é preconizado como promoção de saúde, com ênfase no cuidado integral vem sendo acompanhada de relatos profissionais referentes a barreiras para sua atuação, o que têm dificultado o pleno exercício da profissão na área da saúde. Dentre essas barreiras encontra-se, por exemplo, a formação acadêmica para a atuação desses profissionais na saúde pública (NEVES RLR e ASSUMPÇÃO LOT, 2017). Considerando a importância do PEF no âmbito das equipes da APS, através de suas intervenções e práticas sobre o estilo de vida da população, o presente estudo tem por objetivo identificar quais as principais barreiras para atuação deste profissional na APS.

\section{MÉTODOS}

Trata-se de um estudo de revisão integrativa da literatura. Para o levantamento dos documentos, primeiramente determinou-se a questão norteadora do estudo: Quais as barreiras para a atuação do profissional de educação física na atenção primária à saúde? A partir das palavras chave da questão norteadora buscou-se identificar os descritores apropriados na lista de Descritores em Ciências da Saúde. A estratégia adotada para busca no portal da Biblioteca Virtual em Saúde (BVS), nas bases Literatura LatinoAmericana e do Caribe em Ciências da Saúde (LILACS) e Scientific Electronic Library Online (SCIELO) foi a associação dos seguintes descritores e de operadores boleanos: saúde pública OR promoção da saúde OR atenção primária à saúde AND prática profissional AND educação física e treinamento.

Foram incluídos na pesquisa apenas os documentos na íntegra, publicados em inglês e português a partir de 2005 uma vez que foi neste ano que o profissional de educação física foi devidamente aceito como profissional de saúde. A busca compreendeu documentos publicados em língua portuguesa e/ou inglesa e foi realizada em dezembro de 2018.

A análise inicial das fontes foi realizada a partir do título de cada uma delas, em seguida os documentos restantes tiveram seu resumo analisados a fim de observar a pertinência do conteúdo para o objetivo da revisão. Todo o processo de seleção dos documentos foi feito por três pesquisadores, de forma independente, todos profissionais de educação física e com, no mínimo, título de especialista. Só foram inclusos no estudo os documentos com parecer favorável por pelo menos dois pesquisadores.

Após a seleção dos documentos os mesmos foram lidos exaustivamente para apreensão e sistematização das informações previamente determinadas (título, autores, ano de publicação, objetivo do estudo, sujeitos, método da pesquisa, barreiras identificadas para atuação do profissional de educação física na atenção primária à saúde e informações complementares que auxiliassem a discussão dos resultados) que foram registradas em uma ficha/instrumento de análise desenvolvida pelos pesquisadores.

Além dos critérios de elegibilidade já mencionados, levando em consideração a escassez de estudos cujo objetivo primário fosse identificar as barreiras para atuação do PEF na APS, foram inclusos nesta revisão artigos que, apresentassem, de alguma forma as dificuldades percebidas pelos PEF em suas atividades na APS. 


\section{RESULTADOS}

A análise inicial realizada a partir do título identificou um total de 67 documentos nas diferentes fontes. Destes, três foram excluídos por apresentarem apenas o resumo, 13 por se tratar de trabalhos de conclusão de curso em diversos níveis de ensino e especialidades e três por serem documentos em duplicata. Os 48 documentos restantes tiveram seu resumo analisados a fim de observar a pertinência do conteúdo para o objetivo da revisão. Nesta segunda análise, 14 artigos foram selecionados para análise na íntegra e compor o total de estudos analisados nesta revisão (Figura 1).

Figura 1 - Flow chart demonstrando o processo de seleção dos artigos que compõe a revisão integrativa.

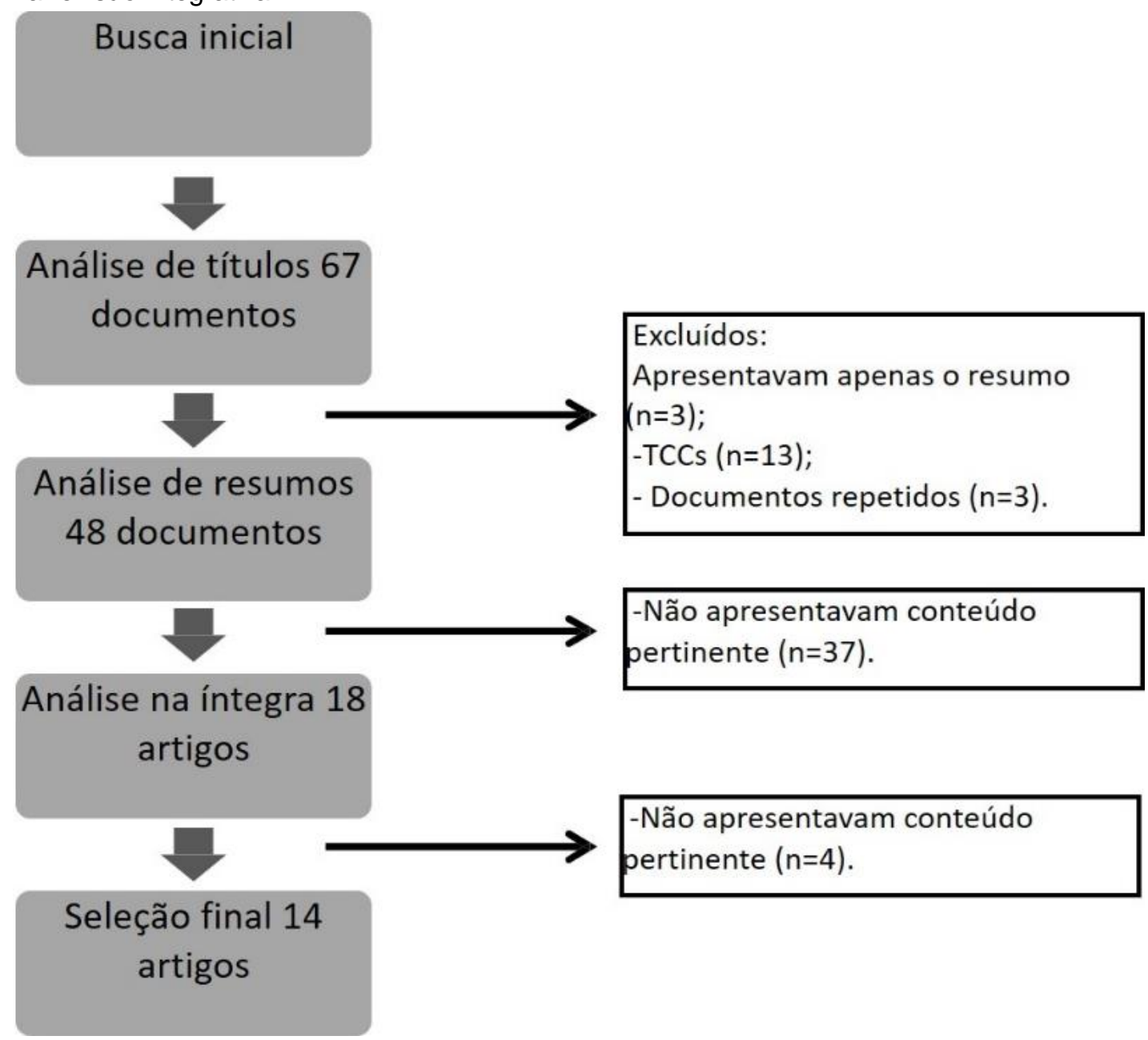

Fonte: Novaes CRMN, et al., 2020.

Considerando os catorze estudos incluídos neste manuscrito, sete são artigos caracterizados como originais, quatro são descritos como revisões e três como análises documentais. De modo geral, os textos relatam a importância do profissional de Educação Física em relação às políticas de saúde pública. Dez desses artigos articulam de alguma forma a formação profissional como uma barreira na atuação do PEF e três apontam a deficiência das estruturas físicas e/ou recursos materiais.

Além destas barreiras destacaram-se ainda a representação social de gestores, profissionais e usuários sobre Educação Física, número reduzido de profissionais atuando na APS e o isolamento desses profissionais dificultando o trabalho interdisciplinar. O quadro 1 detalha os estudos analisados nesta revisão e organiza os principais achados relacionados às barreiras para a atuação do profissional de educação física na atenção primária à saúde (Quadro 1). 


\section{Revista Eletrônica Acervo Saúde / Electronic Journal Collection Health | ISSN 2178-2091}

Quadro 1 - Síntese dos objetivos, método e resultados referentes às barreiras para a atuação do Profissional de Educação Física na atenção básica.

\begin{tabular}{|c|c|c|c|}
\hline Autor/ ano & Objetivo & Método & $\begin{array}{c}\text { Resultados } \\
\text { (Quanto às barreiras para atuação do PEF na } A B \text { ) }\end{array}$ \\
\hline $\begin{array}{l}\text { Anjos TC e } \\
\text { Duarte ACG } \\
\text { (2009). }\end{array}$ & $\begin{array}{l}\text { Pesquisar currículos de Graduação em } \\
\text { Educação Física visando a analisar: i) o } \\
\text { objeto dos cursos; ii) quais as } \\
\text { disciplinas relacionadas à saúde; iii) a } \\
\text { existência das disciplinas de Saúde } \\
\text { Coletiva e Saúde Pública; e iv) a } \\
\text { existência de disciplinas de estágio em } \\
\text { saúde. }\end{array}$ & $\begin{array}{l}\text { Análise do Departamento de Educação Física da UFSCar } \\
\text { ocorreu com investigação de currículos de instituições, de modo } \\
\text { indireto, através da consulta de material nos sites. }\end{array}$ & $\begin{array}{l}\text { O descompasso entre o que propõem as tendências em saúde } \\
\text { para os serviços públicos de saúde e a formação do PEF. Além } \\
\text { do reconhecimento constitucional tardio e a atuação limitada } \\
\text { exclusivamente ao núcleo de conhecimento desse profissional. }\end{array}$ \\
\hline $\begin{array}{l}\text { Carvalho } \\
\text { FFB (2009). }\end{array}$ & $\begin{array}{l}\text { Analisar a Carta Brasileira de } \\
\text { Prevenção Integrada na Área da Saúde } \\
\text { na perspectiva da Educação Física, } \\
\text { documento redigido pelo CONFEF em } \\
\text { 2006, a partir do enfoque radical da } \\
\text { Promoção da Saúde. }\end{array}$ & $\begin{array}{c}\text { Abordagem qualitativa, com análise documental da Carta } \\
\text { Brasileira de Prevenção Integrada na Área da Saúde na } \\
\text { perspectiva da Educação Física, documento redigido pelo } \\
\text { CONFEF em 2006, a partir do enfoque radical da Promoção da } \\
\text { Saúde, como denominado por Buss (2007). }\end{array}$ & $\begin{array}{l}\text { Não apresenta explicitamente as dificuldades para atuação, } \\
\text { porém, questiona o documento que deveria nortear os } \\
\text { profissionais para atuação na área da saúde, mas, que, no } \\
\text { entanto, acaba por biologizar e medicalizar a Educação Física em } \\
\text { sua relação com a sociedade, não relacionando a atividade física } \\
\text { e a saúde com questões sociais mais amplas que impedem que } \\
\text { os sujeitos tomem decisões mais saudáveis, tais como os } \\
\text { condicionantes econômicos, culturais, étnicos e políticos. }\end{array}$ \\
\hline $\begin{array}{l}\text { Silva ALF, et } \\
\text { al. (2009). }\end{array}$ & $\begin{array}{l}\text { Refletir sobre a atuação do profissional } \\
\text { de Educação Física na Estratégia } \\
\text { Saúde de Família de Sobral, inserido } \\
\text { no processo de formação da } \\
\text { Residência Multiprofissional em Saúde } \\
\text { da Família }\end{array}$ & $\begin{array}{l}\text { Relato a partir das Experiências vivenciadas, das reflexões } \\
\text { coletivas, e das contribuições do PEF na melhoria das } \\
\text { condições de saúde e da qualidade de vida da população. }\end{array}$ & $\begin{array}{c}\text { A grande demanda do serviço, a diferença dos horários de } \\
\text { trabalhos, grupos organizados a partir de doenças, privilegiar } \\
\text { como atividade física apenas caminhada e ginástica, limitam a } \\
\text { participação da comunidade e demais profissionais nas práticas e } \\
\text { promoção da atividade física. }\end{array}$ \\
\hline $\begin{array}{l}\text { Andrade DR, } \\
\text { et al. (2012). }\end{array}$ & $\begin{array}{l}\text { Descrever as ações de promoção da } \\
\text { atividade física da pesquisa "Ambiente } \\
\text { Ativo" }\end{array}$ & $\begin{array}{l}\text { Participaram do estudo adultos e idosos sendo } 226 \text { Usuários e } \\
30 \text { ACS do SUS sendo dividido em quatro etapas: o diagnóstico, } \\
\text { intervenção de educação em saúde, intervenção dos ACS como } \\
\text { promotor da atividade em adultos atendidos pela estratégia } \\
\text { Saúde da família e Avaliação da rede de promoção da atividade } \\
\text { física no distrito. }\end{array}$ & $\begin{array}{l}\text { Deficiências nas estruturas físicas, ausência de áreas verdes, de } \\
\text { saneamento básico, de segurança noturna e no trânsito são as } \\
\text { principais, seguidas pela ausência de convites por conhecidos, } \\
\text { eventos esportivos e áreas de lazer próximo da residência. }\end{array}$ \\
\hline
\end{tabular}

REAS/EJCH | Vol.12(11) | e4857 | DOI: https://doi.org/10.25248/reas.e4857.2020 Página $\mathbf{5}$ de $\mathbf{1 1}$ 


\begin{tabular}{|c|c|c|c|}
\hline $\begin{array}{l}\text { Scabar TG, } \\
\text { et al. (2012). }\end{array}$ & $\begin{array}{l}\text { Discutir a atuação do profissional da EF } \\
\text { no Sistema Único de Saúde (SUS) com } \\
\text { vistas à promoção da saúde, a partir da } \\
\text { literatura. }\end{array}$ & $\begin{array}{l}\text { Revisão de literatura durante o período de março a dezembro } \\
\text { de } 2010 \text { realizou a análise dos seguintes pontos: Perfil } \\
\text { profissional do educador físico; Saúde Pública, Promoção da } \\
\text { Saúde e Educação em Saúde; Atenção Primária à Saúde (APS) } \\
\text { e Atenção Básica no Sistema Único de Saúde (SUS); Estratégia } \\
\text { Saúde da Família (ESF); Núcleos de Apoio à Estratégia Saúde } \\
\text { da Família (NASF) e A atuação do educador físico no NASF. }\end{array}$ & $\begin{array}{l}\text { A formação profissional com parâmetros puramente biológicos, } \\
\text { distanciando o PEF da demanda imposta pelos serviços públicos } \\
\text { de saúde; A pouca divulgação sobre a viabilidade das propostas } \\
\text { da PNPS nesse processo de formação;Escassez de estudos } \\
\text { sobre a atuação dos PEFs no SUS e do incentivo à } \\
\text { implementação das ideias da promoção da saúde na área. }\end{array}$ \\
\hline $\begin{array}{l}\text { Ferreira SE, } \\
\text { et al. (2013). }\end{array}$ & $\begin{array}{l}\text { Apresentar o curso de Bacharelado em } \\
\text { Educação Física - Modalidade Saúde } \\
\text { da Universidade Federal de São Paulo } \\
\text { - Campus Baixada Santista. }\end{array}$ & $\begin{array}{l}\text { Fazendo uso do Projeto Político Pedagógico do Campus PPP } \\
13 \text { e o Plano de Desenvolvimento Institucional de 2005. Traça } \\
\text { um histórico da formação em Educação Física no Brasil, e } \\
\text { especificação do curso ofertado pela UNIFESP-BS. }\end{array}$ & $\begin{array}{l}\text { A inconsistência entre a formação profissional em EF com sua } \\
\text { atuação nos serviços de saúde, tornar frágil essa relação. }\end{array}$ \\
\hline $\begin{array}{l}\text { Correa LQ, } \\
\text { et al. (2014). }\end{array}$ & $\begin{array}{l}\text { Apresentar as atividades de } \\
\text { competência e atuação da Educação } \\
\text { Física nas residências } \\
\text { multiprofissionais em saúde e as } \\
\text { dificuldades dessa experiência. }\end{array}$ & $\begin{array}{l}\text { O texto não deixa claro o método usado. Mas aponta como } \\
\text { sujeitos os Residentes da universidade de Rio Grande: } \\
\text { Residentes dos programas em Residência Integrada } \\
\text { Multiprofissional Hospitalar com Ênfase na Atenção à Saúde } \\
\text { Cardiometabólica do Adulto (RIMHAS) e da Residência } \\
\text { Multiprofissional em Saúde da Família (RMSF). }\end{array}$ & $\begin{array}{l}\text { A formação ainda fortemente disciplinar e calcada em regimes } \\
\text { profissionais e de competências, as disputas particulares da } \\
\text { Educação Física, a incapacidade do SUS de ser efetivado, as } \\
\text { dificuldades de avançar em trabalho coletivo e a gestão política. }\end{array}$ \\
\hline $\begin{array}{l}\text { Neves RLR, } \\
\text { et al. (2015). }\end{array}$ & $\begin{array}{c}\text { Refletir sobre a produção acadêmica } \\
\text { brasileira entre os anos } 2000 \text { e } 2012 \text {, } \\
\text { na temática Educação Física na Saúde } \\
\text { Pública. }\end{array}$ & $\begin{array}{c}\text { Revisão bibliográfica de } 60 \text { textos publicados em periódicos } \\
\text { científicos, livros e capítulos de livros, teses e dissertações } \\
\text { compilados na base de dados "Teses e dissertações" da } \\
\text { CAPES e no "Google acadêmico". }\end{array}$ & $\begin{array}{c}\text { Reafirma a importância do professor de Educação Física em } \\
\text { relação às políticas de saúde; a representação social de gestores, } \\
\text { profissionais e usuários sobre Educação Física; a questão da } \\
\text { formação profissional; e a abordagem de experiências em } \\
\text { unidades de saúde diversas. }\end{array}$ \\
\hline $\begin{array}{l}\text { Rodrigues } \\
\text { JD, et al. } \\
\text { (2015). }\end{array}$ & $\begin{array}{l}\text { Descrever o perfil e aspectos da } \\
\text { atuação dos Profissionais de Educação } \\
\text { Física (PEF) nos Núcleos de Apoio à } \\
\text { Saúde da Família (NASF) da região } \\
\text { metropolitana de João Pessoa, PB. }\end{array}$ & $\begin{array}{c}\text { Descritivo de natureza quantitativa realizou entrevista } \\
\text { semiestruturada com } 15 \mathrm{PEF} \text {, que atuavam há pelo menos seis } \\
\text { meses nos NASF da região metropolitana. }\end{array}$ & Relata como única dificuldade a falta de trabalho intersetorial. \\
\hline $\begin{array}{l}\text { Nascimento } \\
\text { PMM e } \\
\text { Oliveira } \\
\text { MRR (2016). }\end{array}$ & $\begin{array}{c}\text { Analisar apontamentos e perspectivas } \\
\text { que contribuem para consolidação de } \\
\text { nova maneira de pensar a formação } \\
\text { em Educação Física para o campo da } \\
\text { saúde, num recorte que explicita as } \\
\text { dimensões política, prática e } \\
\text { pedagógicas concernentes a esse } \\
\text { processo formativo. }\end{array}$ & $\begin{array}{c}\text { Bibliográfico, de natureza conceitual identifica as contribuições } \\
\text { de um grupo de autores, com forte afinidade com a área das } \\
\text { ciências humanas e sociais, vinculado tanto ao campo da saúde } \\
\text { quanto ao campo da EF, para uma renovação curricular da } \\
\text { formação dessa área. }\end{array}$ & A formação acadêmica. \\
\hline
\end{tabular}

REAS/EJCH | Vol.12(11) | e4857 | DOI: https://doi.org/10.25248/reas.e4857.2020 Página 6 de 11 


\begin{tabular}{|c|c|c|c|}
\hline $\begin{array}{l}\text { Saporetti } \\
\text { GM, et al. } \\
\text { (2016). }\end{array}$ & $\begin{array}{l}\text { Analisar as ações de Promoção da } \\
\text { Saúde realizadas pelos PEFs nos } \\
\text { NASFs em Minas Gerais. }\end{array}$ & $\begin{array}{l}\text { Pesquisa de abordagem qualitativa e exploratória realizou a } \\
\text { partir de análise descritiva dos documentos produzidos pelo } \\
\text { Conselho Federal de Educação Física a partir de } 1998 \text { (no que } \\
\text { se refere à definição e regulação das atividades do PEF, com } \\
\text { ênfase na APS), dois grupos focais com a participação de PEFs } \\
\text { matriculados no curso de especialização em atenção básica em } \\
\text { saúde da família (CEABSF) da UFMG que estavam inseridos } \\
\text { nos NASFs em Minas Gerais e a presença de um moderador e } \\
\text { um observador. Depois de gravadas em áudio, as falas foram } \\
\text { transcritas, usando técnica de análise de conteúdo de Bardin. }\end{array}$ & $\begin{array}{l}\text { A falta de infraestrutura física, de materiais e equipamentos; A } \\
\text { localização, os horários das atividades ofertadas e a violência da } \\
\text { região; A baixa adesão de crianças, adolescentes e homens nos } \\
\text { grupos e dificuldade de mudança do paradigma de atendimento } \\
\text { curativo; O reduzido número de PEFs atuando no NASF e a } \\
\text { necessidade de capacitá-los para trabalhar na atenção primária; } \\
\text { O desconhecimento ou não entendimento de seu papel pelos } \\
\text { demais profissionais; Além disso a ausência de transporte para o } \\
\text { deslocamento entre os polos, a cobrança por produtividade, a } \\
\text { impossibilidade de acompanhamento do tratamento do paciente e, } \\
\text { por fim, a solidão advinda da ausência de outro PEF para } \\
\text { compartilhamento do trabalho foram outros itens destacados. }\end{array}$ \\
\hline $\begin{array}{l}\text { Carvalho } \\
\text { AS, et al. } \\
(2017)\end{array}$ & $\begin{array}{c}\text { Descrever e analisar os tipos de } \\
\text { atuação do profissional de Educação } \\
\text { Física no Sistema Único de Saúde } \\
\text { (SUS). }\end{array}$ & $\begin{array}{c}\text { Revisão sistemática da literatura realizada nas bases de dados } \\
\text { Google Acadêmico e Scielo. Analisou publicações entre os anos } \\
\text { de } 2005 \text { e } 2016 \text { por meio dos descritores: Sistema Único de } \\
\text { Saúde, Atividade Física e Educação Física. Após a seleção } \\
\text { inicial, realizaram-se análises dos títulos, resumos e textos } \\
\text { completos. }\end{array}$ & $\begin{array}{c}\text { A matriz curricular deficitária quando se trata de Saúde Pública e } \\
\text { Saúde Coletiva, com poucas intervenções profissionais na área } \\
\text { da saúde e com uma perspectiva biologista e /ou clássica voltada } \\
\text { para o trabalho individual e o espaço privado. Além disso, a } \\
\text { prática do PEF ainda não é bem consolidada e definida no SUS, } \\
\text { sem uma demanda específica definida, as equipes de saúde das } \\
\text { UBS ainda ignoram o potencial de atuação do núcleo da } \\
\text { Educação Física na prevenção, promoção, atenção ou } \\
\text { reabilitação da saúde. }\end{array}$ \\
\hline $\begin{array}{l}\text { Neves RLR } \\
\quad \text { e } \\
\text { Assumpção } \\
\text { LOT (2017). }\end{array}$ & $\begin{array}{c}\text { Analisar as percepções de profissionais } \\
\text { de Educação Física sobre os } \\
\text { processos de formação e seus reflexos } \\
\text { na atuação em serviços da Saúde } \\
\text { Pública em Goiânia/GO. }\end{array}$ & $\begin{array}{l}\text { Pesquisa qualitativa exploratória realizou entrevista } \\
\text { semiestruturada com nove trabalhadores de quatro instituicões } \\
\text { da Secretaria Municipal de Saúde de Goiânia. As entrevistas } \\
\text { duraram em média uma hora e dez minutos e foram realizadas } \\
\text { individualmente em ambientes restritos dentro das quatro } \\
\text { unidades de saúde. }\end{array}$ & $\begin{array}{l}\text { Segundo os profissionais são a formação inicial: tradicional e } \\
\text { tecnicista; mais centrada no campo da iniciação esportiva, } \\
\text { atividade física, exercícios físicos, escola/ didática e da academia } \\
\text { de ginástica; e pouco focada nas necessidades dos serviços } \\
\text { públicos de saúde, gerando fragilidade nesta dimensão da } \\
\text { intervenção profissional. }\end{array}$ \\
\hline $\begin{array}{l}\text { Santos TI, et } \\
\text { al. (2017). }\end{array}$ & $\begin{array}{l}\text { Analisar os fatores associados às } \\
\text { práticas de Apoio Matricial realizadas } \\
\text { pelos Profissionais de Educação Física } \\
\text { do Núcleo de Apoio à Saúde da Família } \\
\text { (NASF) no Brasil e segundo as suas } \\
\text { regiões. }\end{array}$ & $\begin{array}{l}\text { Estudo exploratório, descritivo e de abordagem quantitativa. } \\
\text { Realizaram entrevistas telefônicas com } 296 \text { profissionais de } \\
\text { Educação Física credenciados nas equipes do NASF no período } \\
\text { de julho e setembro de } 2011, \text { o roteiro das entrevistas foi } \\
\text { composto por } 59 \text { questões referentes às características } \\
\text { sociodemográficas, condições de trabalho e as atividades de } \\
\text { apoio matricial desenvolvidas por esses profissionais. }\end{array}$ & $\begin{array}{l}\text { Divergências na atuação dos profissionais de Educação física do } \\
\text { NASF, devido a múltiplos fatores nas condições de trabalho e a } \\
\text { formação profissional, entre eles: o apoio da gestão em saúde, a } \\
\text { prática de acolhimento, investimentos na qualificação profissional, } \\
\text { disponibilidade de recursos materiais, integração do PEF à equipe } \\
\text { NASF e o desempenho das atividades de Apoio Matricial. }\end{array}$ \\
\hline
\end{tabular}

Fonte: Novaes CRMN, et al., 2020.

REAS/EJCH | Vol.12(11) | e4857 | DOI: https://doi.org/10.25248/reas.e4857.2020 Página 7 de 11 


\section{DISCUSSÃO}

O fenômeno de transição epidemiológica ocorrido nas últimas décadas vem indicando a necessidade da garantia de políticas públicas de incentivo à prática da atividade física sistemática, bem como de estímulo a outros hábitos saudáveis para a população, o que demonstra a relevância do trabalho do profissional de educação física (OLIVEIRA DCR, et al., 2016).

Neste contexto, os resultados do presente estudo apresentam algumas das diversas barreiras encontradas por estes profissionais para a operacionalização das ações de promoção das práticas corporais/atividade física no âmbito do NASF AB e/ou do Programa Academias da Saúde.

Como achado mais prevalente nos estudos analisados, diversos autores apontam a fragilidade e a inconsistência na formação inicial dos PEFs para atuação na saúde coletiva e/ou atenção primária à saúde nos cursos de graduação. A formação tradicional centrada no tecnicismo, mais voltada para o campo da iniciação esportiva, pouco focada nas necessidades dos serviços públicos de saúde e dos territórios não prepara o profissional para atuar frente à resolução dos problemas de saúde da população (CARVALHO AS, et al., 2017; NEVES RL e ASSUMPÇÃO LOT, 2017, FERREIRA SE, et al., 2012; ANJOS TC e DUARTE ACG, 2009).

Ainda com vistas à formação, estudos fazem referência aos poucos currículos nos curso de formação profissional da área que ofertam disciplinas de Saúde Pública e Saúde Coletiva e que incluem intervenções profissionais no âmbito da saúde, e que quando as mesmas são ofertadas, ocorrem ainda de forma tímida, limitada, sob enfoque do modelo biologista e individualista (CARVALHO AS, et al., 2017; ANJOS TC e DUARTE ACG, 2009; SAPORETTI GM, et al., 2016).

Esta realidade foi corroborada pelos desfechos de estudos realizados por Costa LC, et al. (2012), cujo objetivo foi analisar as grades curriculares de 61 cursos de Educação Física e constatou que 44 deles não ofertava nenhuma disciplina relacionada à Saúde Pública/Saúde Coletiva.

Em contraponto, Pasquim HM (2010) afirma que mesmo fazendo parte do componente curricular dos cursos da área em questão, a Saúde Coletiva ainda é marginalizada na formação em Educação Física e não apresenta práticas acadêmicas consistentes que permitam o desenvolvimento efetivo e consistente das práticas profissionais neste contexto. Loch RM, et al. (2020) concordam com esta afirmação quando reportam que não se oportuniza aproximação concreta dos discentes com o campo da saúde coletiva somente com a oferta de disciplinas específicas.

Os autores sugerem que se faz necessário ir além disso, enfocando perspectivas de ensino e possibilidades de aprendizagem mais ampliadas, propiciadas pelo envolvimento com os serviços de saúde (integração ensino-serviço), e que tais experiências possam ser oportunizadas ainda nos primeiros semestres do curso. Considerando a formação ainda precária para estas experiências e a necessidade iminente pela busca do desenvolvimento de competências específicas para a atuação na APS, Falci DM e Belisário AS (2013) citam a pós-graduação como uma opção para a superação dessa defasagem na formação inicial do PEF.

Atualmente, algumas características da atuação dos PEF podem ser consideradas consequências desse modelo de formação desarticulado da necessidade social, como a organização de grupos a partir das doenças e o privilégio de apenas caminhada e ginástica como possibilidades de práticas corporais, limitando assim o acesso da comunidade à promoção de atividade físicas/práticas corporais diversas (SILVA ALF, et al., 2009).

Brugnerotto $F$ e Simões $R$ (2009) afirmam que um conceito de saúde reducionista concebido pela instituição durante a construção curricular dos cursos de formação em Educação Física favorece a ideia de cabe ao PEF prescrever e monitorar a prática de atividades físicas considerando os aspectos biológicos do indivíduo esquecendo-se dos outros fatores correlacionados. É perceptível que, como ainda ocorre em outras áreas da saúde, a formação em Educação Física ainda apresenta caráter uniprofissional, com poucas ou inexistentes oportunidades de compartilhamento de saberes e de colaboração através de práticas interprofissionais (LOCH RM, et al., 2020). 
Estudo realizado por Oliveira BN e Watchs $F$ (2019) destaca que mesmo diante da complexidade existente nos cenários e nas práticas que envolvem a atuação dos PEF na APS, percebe-se uma atuação reducionista, com ênfase na aplicação de protocolos universais.

Guarda FRB (2014) destaca um descompasso entre formação e as necessidades do mercado de trabalho, alertando que isso é observado em todas as profissões da saúde, inclusive na Educação Física. O autor atribui esse desalinhamento à fragmentação do conhecimento, enfoque no caráter biológico e reprodução de práticas centradas na doença, e na reprodução de protocolos e procedimentos pré-definidos.

O Ministério da Saúde apresenta uma ampliada compreensão em relação à atuação do PEF na atenção primária à saúde, tendo em vista sua recente valorização e inserção no campo da saúde como um importante agente promotor de saúde frente às políticas públicas neste setor (SCABAR TC, et al., 2012).

Em contraponto a esta afirmação, os resultados de estudo realizado por Saporetti GM, et al.,(2016) indicam que, além de existir uma falta de conhecimento dos demais profissionais de saúde sobre o papel do PEF bem como de suas potencialidades sua prática ainda não é considerada bem consolidada e definida no SUS, ignorando-se assim o seu potencial de atuação na prevenção, promoção assistência ou reabilitação da saúde, o que sugere a necessidade de elaboração de iniciativas técnico-pedagógicas que estimulem discussões entre os profissionais e que fortaleçam as práticas em saúde integradas, não apenas no campo da educação física, mas em todas as áreas que compõem a atenção primária à saúde (CARVALHO AS, et al., 2017).

Além destas percepções, estes registros reforçam a escassez de estudos sobre a atuação dos PEFs no SUS, e para além disso, revelam a necessidade do incentivo à implementação das ideias da promoção da saúde na área (FERREIRA SE, et al., 2012).

No que diz respeito ao ambientes destinados à promoção da atividade física na APS e à sua infraestrutura, os resultados desse estudo ainda trazem como barreiras significativas para atuação do PEF alguns aspectos relacionados à falta de estrutura ou inexistência de espaços públicos adequados destinados às práticas corporais, presença de esgoto a céu aberto e de lixo nas vias e na imediações dos espaços, violência, insegurança no trânsito (SAPORETTI GM, et al., 2016; ANDRADE DR, et al., 2013).

Tais fatores dificultam a adesão dos usuários e descontinuam o cuidado longitudinal em saúde, uma vez que impedem ou desestimulam o usuário de se deslocar até esses espaços e/ou de se manterem assíduos. Hino AFF, et al. (2010) reportam fortes evidências de que o ambiente onde as pessoas vivem podem influenciar significativamente na prática de atividade física da população.

Neste mesmo sentido, Silva MC, et al. (2012) constatam que, para a população que não tem acesso a espaços privados, a estrutura dos espaços públicos destinados à atividade física são determinantes para aumentar o número de pessoas fisicamente inativas.

Ao analisar a associação da prática de atividade física com a percepção do ambiente comunitário por adultos, um estudo identificou a importância de se investir em ambientes que estimulem o convívio das pessoas para proporcionar oportunidades para a criação de redes sociais importantes para a prática de atividade física. A referida pesquisa identificou que a presença de clubes próximos a residências foi associada positivamente com a prática de atividade física no lazer. O mesmo estudo ressalta que se faz necessário a existência de investimentos em segurança pública e em ambientes e instalações favoráveis à promoção da atividade física em regiões de maior desigualdade social. (FLORINDO AA, et al., 2011)

Com vistas às fragilidades que influenciam no processo de gestão do trabalho do PEF, apresentam-se a ausência de transporte para deslocamento entre as unidades básicas do território, a cobrança por produtividade, a impossibilidade de acompanhamento longitudinal do tratamento dos usuários e a solidão advinda da ausência de outro PEF para o compartilhamento de atribuições e experiências (SAPORETTI GM, et al., 2016). Silva e colaboradores (2009) citam ainda a grande demanda de serviços e a diferença dos horários das atividades do PEF e do funcionamento normal da UBS.

Outros fatores foram elencados em menor escala, porém merecem ser destacados pela sua relevância no processo de trabalho do PEF na atenção básica, como a falta de apoio da gestão em relação à disponibilização de recursos materiais e à oferta de formação continuada. 
Neste contexto e baseado na premissa de que a atuação de qualidade do PEF está relacionada a múltiplos fatores, Santos TI, et al. (2017) destacam que o apoio da gestão no tocante à disponibilidade de estrutura física e material, além de acolhimento pedagógico são fatores que se correlacionam positivamente com a realização de apoio matricial (e consequentemente de seus componentes: educação permanente, educação em saúde, controle social e ampliação da clínica), e que investir na qualificação dos profissionais minimiza as discrepâncias na atuação do PEF que atuam na atenção básica nas diferentes regiões do país.

Reforçando esta ideia, um estudo realizado por Santos SFS, et al. (2017) que teve o objetivo de analisar os fatores associados às práticas de apoio matricial do PEF no NASF no Brasil identificou que o suporte pedagógico aos profissionais e a disponibilização dos recursos materiais por parte da gestão são aspectos positivos que contribuem para o melhor desempenho destes profissionais no processo de trabalho.

As constatações provenientes do presente estudo podem contribuir para ampliação do debate a respeito da formação do PEF para a atuação na atenção primária à saúde e fortalecer as ações de gestão dos programas nos quais este profissionais atuam, favorecendo a compreensão sobre as principais dificuldades enfrentadas pelo público em questão e possibilitando o redirecionamento de práticas acadêmicas e de gestão das políticas públicas no âmbito das práticas corporais/atividade física nos cenários que os envolvem, fortalecendo ainda a atuação do PEF neste nível de atenção à saúde, e proporcionando maiores oportunidades de resposta às demandas sociais no âmbito da promoção da atividade física.

\section{CONSIDERAÇÕES FINAIS}

Os resultados encontrados apontam que algumas das barreiras para atuação do profissional de educação física na atenção básica estão relacionadas à gestão dos programas onde esses profissionais atuam, a exemplo da deficiência da estrutura física dos locais de trabalho, da escassez de recursos materiais e do baixo número de profissionais em atuação para uma população adscrita numerosa nos territórios. Apresenta ainda as barreiras relacionadas à formação deste profissional, como a dificuldade no trabalho interdisciplinar, a formação voltada para o âmbito esportivo e a escassez ou inexistência de disciplinas e experiências acadêmicas em saúde coletiva.

\section{REFERÊNCIAS}

1. ANDRADE DR, et al. Do diagnóstico à ação: A experiência da pesquisa Ambiente Ativo na promoção da atividade física em Ermelino Matarazzo, na zona leste de São Paulo, SP. Rev Bras Ativ Fís Saúde, 2013; 17(3):235-8

2. ANJOS TC, DUARTE ACG. A Educação Física e a Estratégia de Saúde da Família. Physis Revista de Saúde Coletiva, 2009; v. 19, n.4, pp.1127-1144.

3. BRASIL. Lei $\mathrm{n}^{\circ} 8.880$ de 19 de setembro de 1990. Dispõe sobre as condições para a promoção, proteção e recuperação da saúde, a organização e o funcionamento dos serviços correspondentes e dá outras providências. Diário Oficial da União, 19 Set 1990.

4. BRASIL. Portaria ํㅜ 154, de 24 de janeiro de 2008. Cria os Núcleos de Apoio à Saúde da Família - NASF. Diário Oficial da União, 24 de Jan 2008.

5. CARVALHO FFB. Análise crítica da Carta Brasileira de Prevenção Integrada na Área da Saúde na Perspectiva da Educação Física através do enfoque radical de promoção da saúde. Saude soc.[Internet]. 2009; (2): 227-236.

6. CARVALHO AS, et al. Atuação do profissional de educação física no sistema único de saúde: revisão sistemática. Rev Bras Promoç Saúde. 2017; 30(3): 1-11.

7. CORREAA LQ, et al. A atuação da educação física nas residências multiprofissionais em saúde. Rev Bras Promoç Saúde. 2014; 27(3): 428-433.

8. COSTA LC, et al. Formação profissional e produtividade em saúde coletiva do profissional de Educação Física. Rev Bras Ativ Fís Saúde [Internet]. 2012; 17(2):107-13.

9. FALCI DM, BELISÁRIO SA. The position of physical education professionals within primary healthcare and the challenges in their training. Interface (Botucatu), 2013; 17(47): 885-99.

10. FERREIRA SE, et al. Formação profissional em Educação Física e saúde na Universidade Federal de São Paulo. Rev Bras Ativ Fis Saúde. 2013; 18: 646-651.

11. FLORINDO AA, et al. Percepção do ambiente e prática de atividade física em adultos residentes em região de baixo nível socioeconômico. Rev Saúde Pública. 2011; 45(2): 302-310.

12. GUARDA FRB, et al. Intervenção do profissional de educação física: formação, perfil e competências para atuar no Programa Academia da Saúde. Rev Pan-Amaz Saude [Internet]. 2014; 5(4): 63-74. 
13. GROPPO HS, et al. Efeitos de um programa de atividade física sobre os sintomas depressivos e a qualidade de vida de idosos com demência de Alzheimer. Rev. bras. Educ. Fís. Esporte. 2012; 26(4): 543-551.

14. HALLAL PC, et al. Avaliação do programa de promoção da atividade física Academia da Cidade de Recife, Pernambuco, Brasil: percepções de usuários e não-usuários. Cad. Saúde Pública. 2010; 1: 70-78.

15. HINO AAF, et al. Ambiente construído e atividade física: uma breve revisão dos métodos de avaliação. Rev. bras. cineantropom. desempenho hum. [Internet]. 2010; 12(5): 387-394.

16. JESUS GM, JESUS EFA. Nível de atividade física e barreiras percebidas para a prática de atividades físicas entre policiais militares. Rev. Bras. Ciênc. Esporte. 2012; 34(2): 433- 438.

17. LEE IM, et al. Lancet Physical Activity Series Working Group: Effect of physical inactivity on major non communicable diseases worldwide: an analysis of burden of disease and life expectancy. Lancet. 2012, 380: 219-29.

18. LOCH MR, et al. A urgência da Saúde Coletiva na formação em Educação Física: Lições com o COVID-19. Cien Saude Colet [internet]. 2020.

19. MATSUDO SM. Atividade física na promoção da saúde e qualidade de vida no envelhecimento. Rev. bras. Educ. Fís. 2006; 20(5): 135-137.

20. MENDONÇA BCA, et al. Do diagnóstico à ação: experiências em promoção da atividade física programa Academia da Cidade Aracaju: promovendo saúde por meio da atividade física. Rev Bras Ativ Fís Saúde. 2009; 14(3): 211-216.

21. NASCIMENTO PMM, OLIVEIRA MRR. Perspectivas e possibilidades para a renovação da formação profissional em educação física no campo da saúde. Pensar a Prática, Goiânia, 2016; $19: 1$.

22. NEVES RLR, et al. Educação Física na saúde pública: Revisão Sistemática. R. bras. Ci. e Mov 2015; $23(2)$ : $163-177$.

23. NEVES RL, ASSUMPÇÃO LOT. Formação e intervenção profissional em saúde pública: percepções de profissionais de educação física. Movimento. 2017; 23(1): 201-212.

24. OLIVEIRA BN, WACHS F. Educação Física e Atenção Primária à Saúde: o apoio matricial no contexto das redes. Rev Bras Ativ Fis Saúde. 2018; 23:e0064.

25. OLIVEIRA DCR, et al. Competências conceituais dos profissionais para intervenção em programas de atividade física na atenção básica. In: BARROS MVG, et al. Pesquisa em estilo de vida e saúde. Recife: Editora Universitária da UFRPE. 2016; 204-212.

26. PASQUIM HM. A saúde coletiva nos cursos de graduação em Educação Física. Saude soc. [Internet]. 2010; 19(1): 193-200.

27. PINTO NRS, et al. Política de saúde e gestão no processo de (re)construção do SUS em município de grande porte: um estudo de caso de São Paulo, Brasil. Cad. Saúde Pública. 2009; 25: 927-938.

28. PITANGA FJG, LESSA I. Prevalência e fatores associados ao sedentarismo no lazer em adultos. Cad. Saúde Pública. 2005; 21(3): 870- 877.

29. RODRIGUES JD, et al. Perfil e atuação do Profissional de Educação Física nos Núcleos de Apoio à Saúde da Família na região metropolitana de João Pessoa, PB. Rev Bras Ativ Fís Saúde 2015; 352-365.

30. SANTOS SFS, et al. Apoio Matricial e a atuação do Profissional de Educação Física do Núcleo de Apoio à Saúde da Família. Rev Bras Ativ Fís Saúde. 2017; 22(1): 54-65.

31. SANTOS TI, et al. Vivências de prática de atividade física para Agentes Comunitários de Saúde. In: FLORINDO AA e ANDRADE DRA. Experiências de promoção da atividade física na Estratégia de Saúde da Família. Florianópolis: FAPESP. 2015; 36-50.

32. SAPORETTI GM, et al. O profissional de educação física e a promoção da saúde em núcleos de apoio à saúde da família. Trab. Educ. Saúde. 2016; 14(2): 523-543.

33. SCABAR TG, et al. Atuação do profissional de Educação Física no Sistema Único de Saúde: uma análise a partir da Política Nacional de Promoção da Saúde e das Diretrizes do Núcleo de Apoio à Saúde da Família - NASF. J Health Sci Inst. 2012; 30(4): 411-418.

34. SILVA ALF, et al. Educação física na atenção primária à saúde em Sobral-Ceará: desenhando saberes e fazeres integralizados. Sanare Rev Políticas Públicas. 2009; 8(2): 63-72.

35. WORLD HEALTH ORGANIZATION. Global: recommendations on physical activity for health. Genebra: WHO; 2010. 